\title{
Changing the Culture of Cholecystectomy
}

\author{
Saxon Connor ${ }^{1} \cdot$ James Garden $^{2}$
}

Published online: 25 August 2015

(C) Société Internationale de Chirurgie 2015

To the Editor,

We read with interest the article by Nijseen et al. [1] which demonstrates that the critical view of safety (CVS) following dissection during cholecystectomy is less likely to be achieved in those patients who go on to develop complications, and highlights the significant variance between surgeon perspective and independent review of CVS success. The endpoint of the study by Nijssen et al. was the success or failure to achieve the CVS. However, we authors would argue that the culture should shift to recognising and analysing the steps that lead to the safe attainment of the CVS rather than attainment of the CVS itself. As Nijssen et al. acknowledge, others have identified that up to $80 \%$ of bile duct injuries occur while the surgeon is attempting to achieve the CVS. In addition, the current study has highlighted that many surgeons believe they have attained the CVS when they have not. Surgeons lose spatial awareness and mistakenly believe they are safely obtaining the critical view when they are not.

We believe that the key to avoiding bile duct injuries is to develop a culture of safety when performing cholecystectomy [2]. This involves understanding and actively acknowledging the steps required to achieve the CVS safely [2]. The four steps include reflecting the fundus of the gallbladder cephalad and laterally, lifting Hartmann's pouch up and over to the base of the segment IV pedicle, use of

Saxon Connor

saxon.connor@cdhb.govt.nz

1 Department of Surgery, Canterbury Health, Christchurch, New Zealand

2 Clinical and Surgical Sciences (Surgery), Universitiy of Edinburgh, Royal Infirmary, Edinburgh, UK
Rouviere's sulcus to ensure identification of boundaries of safe dissection and releasing posterior peritoneum prior to performing anterior peritoneal dissection. Crucially, it also has a stop point whereby it is recognised that it is unsafe to proceed with further dissection if these steps cannot be safely undertaken. If the stop point is reached, knowledge and implementation of safe alternatives are required. Such alternatives include conversion to open surgery by the appropriately trained surgeon, completing subtotal cholecystectomy or calling for an experienced hepatobiliary surgeon. If these steps are unattainable, the operation should be abandoned with the aim of referring to such an experienced surgeon. In such situations, fundus first dissection whether open or laparoscopically can only proceed when full consideration of the proximity, involvement and potential risk of injury to the right portal pedicle is given. In these situations, striving to achieve the CVS will exacerbate the risk of bile duct injury. It is only by changing the culture and understanding these steps that BDI will be eliminated. Simply improving surgeon's recognition of the CVS will not change the status quo.

\section{References}

1. Nijssen MAJ, Schreinmakers JMJ, Meyer Z, van der Schelling GP, Colla RMPH, Rijken AM (2015) Complications after laparoscopic cholecystectomy: a video evaluation study of whether the critical view of safety was reached. World J Surg 39:1798-1803. doi:10. 1007/s00268-015-2993-9

2. Connor SJ, Perry W, Nathanson L, Hugh TJ, Hugh TB (2014) Using a standardised method for laparoscopic cholecystectomy to create a concept operation-specific checklist. HPB 16(5):422-429 